\title{
Myocardial TI mapping: where are we now and where are we going?
}

This article was published in the following Dove Press journal:

Research Reports in Clinical Cardiology

26 November 2014

Number of times this article has been viewed

\author{
Amna Abdel-Gadir ${ }^{1,2}$ \\ Thomas A Treibel 1,2 \\ James C Moon ${ }^{1,2}$ \\ 'The Heart Hospital Imaging \\ Centre, University College London \\ Hospitals, London, UK; ${ }^{2}$ Institute of \\ Cardiovascular Science, University \\ College London, London, UK
}

Correspondence: James C Moon

The Heart Hospital Imaging Centre,

University College London Hospitals,

16-18 Westmoreland Street,

London, WIG 8PH, UK

Tel +44203456 308I

Fax +442034563086

Email j.moon@ucl.ac.uk
Abstract: Cardiovascular magnetic resonance offers noninvasive myocardial tissue characterization as a key unique strength over other imaging techniques. In particular T1, a tissue property that alters with disease, has gained prominence as a diagnostic tool. Prior to the administration of contrast, the native $\mathrm{T} 1$ changes with a number of processes such as fibrosis, edema, and infiltration. If a post-contrast scan is also acquired, the post-contrast T1 and extracellular volume fraction can be measured. Detecting and quantifying early and established myocardial pathological processes permits better diagnosis, prognostication, and tracking of therapy.

Keywords: extracellular volume fraction, diffuse fibrosis, interstitium, cardiac remodeling, myocardial intracellular volume

\section{Introduction}

Cardiovascular magnetic resonance (CMR) is an essential diagnostic tool that is well recognized as the gold-standard method of assessing cardiac volumes, function, and mass, with evidence accumulating for its use in ischemia testing. ${ }^{1,2}$ These incremental advantages are complemented by a unique ability to perform in vivo myocardial tissue characterization. The major technique is late gadolinium enhancement (LGE) imaging, also recognized as scar imaging, which detects focal myocardial fibrosis after intravenous administration of gadolinium-based contrast agents when there is a slower washout of contrast in tissues with an increased extracellular space. Initially performed in myocardial infarction, LGE imaging is now recognized in most cardiac diseases. $^{3-7}$ Typical patterns of enhancement occur which are disease specific, aiding diagnosis, while the scar burden is prognostic and helps treatment choices. ${ }^{8}{ }^{8}$ However, an important limitation of LGE imaging is that it is a "difference test" - it compares one area to another. If the whole myocardium is abnormal, this is missed. Key processes, particularly diffuse fibrosis, but also amyloid, fat, and iron deposition, may be missed, particularly in the early phase. This triggered the development of a new field in CMR: direct quantification of signal from the myocardium via parametric mapping techniques. These techniques - T1, T2, and T2* mapping, but particularly T1 mapping - have the potential to become powerful new clinical and research techniques, helping develop and guide therapy to improve patient outcomes. All tissues have natural tissue-specific longitudinal (T1) and transverse (T2) relaxation, as well as dephasing (T2*) times. These may be altered in disease. Mapping techniques measure these and display the actual values in color on a pixel-by-pixel basis for regional, and between-patient, comparisons without the need for post-processing (Figure 1A). When T1 mapping is 

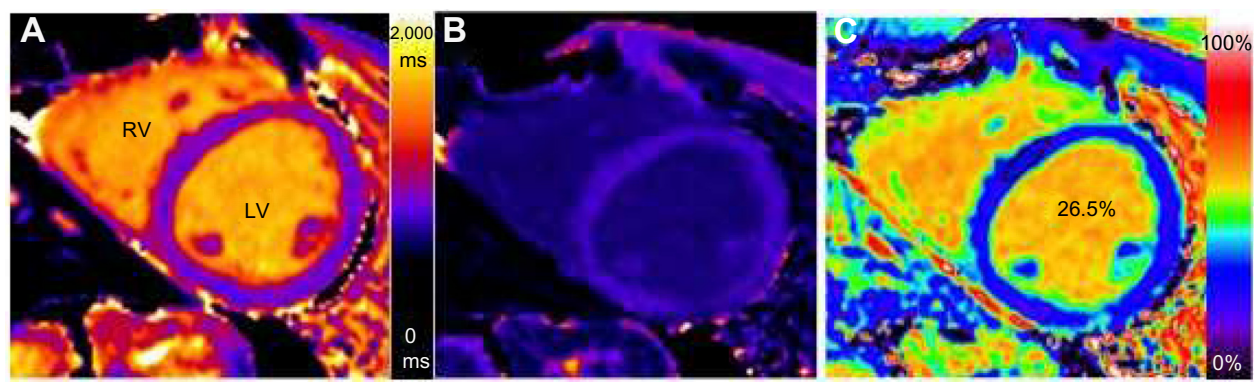

Figure I TI mapping in a healthy volunteer.

Notes: (A) Native TI MOLLI map (myocardial TI I,0I0 ms). (B) Post-contrast TI MOLLI map (myocardial TI $6 I 5$ ms). (C) ECV map (ECV =26.5\%). Abbreviations: ECV, extracellular volume; LV, left ventricle; MOLLI, modified Look-Locker inversion recovery; RV, right ventricle.

used with a standard, gadolinium-based extracellular contrast agent, the extracellular volume (ECV) can be measured (Figure $1 \mathrm{~B}$ and $\mathrm{C}$ ), reflecting interstitial disease, particularly fibrosis, but also edema and amyloid. Early work suggests that ECV measurements may be as prognostically important as left ventricular ejection fraction. ${ }^{10}$ The formation of the T1 Mapping Group and development of the T1 mapping consensus statement reflect the exponential interest, both clinical and academic, in T1 mapping globally. ${ }^{11}$

\section{The development of T I mapping}

$\mathrm{T} 1$, also known as the spin-lattice relaxation time, is an intrinsic magnetic property of tissue that represents longitudinal recovery time of hydrogen atoms after excitation. Each tissue has its own characteristic range of values (expressed in milliseconds) at a selected magnetic field strength, and deviation from these values is used to quantify effects of pathological processes. ${ }^{12}$ Some pathologies (fat, iron, amyloid) change T1 substantially; others (fibrosis) less so, but in a still-measurable way. Standard extracellular contrast agents also change T1 - but as contrast is confined to the interstitium, the ECV can be measured, which provides an additional test by which to assess fibrosis. T1 measurement has improved substantially from the early days of multi-breath-hold fast low-angle shot imaging (FLASH) or Look-Locker sequences, and T1 mapping sequences have been developed by all CMR scanner manufacturers. Incremental improvements to the original modified Look-Locker inversion recovery ([MOLLI] Figure 2A) sequence have occurred with shorter breath holds using the shortened MOLLI sequence ([ShMOLLI] Figure 2B), which requires an acquisition time over nine heartbeats. ${ }^{13-15}$ Newer T1 mapping sequences in development include the saturation recovery single-shot acquisition ([SASHA] Figure 2C) and saturation pulse prepared heart-rate-independent inversion recovery (SAPPHIRE), which use saturation pulses or a combination of saturation and inversion pulses, respectively. ${ }^{16}$

Native T1 and post-contrast maps may be combined to create ECV maps wherein pixel values represent the interstitial volume (Figure 1). ${ }^{17,18}$ The result is expected to have increasing clinical utility - particularly, improved precision and reproducibility (although accuracy and a truth standard are less certain). MOLLI and ShMOLLI

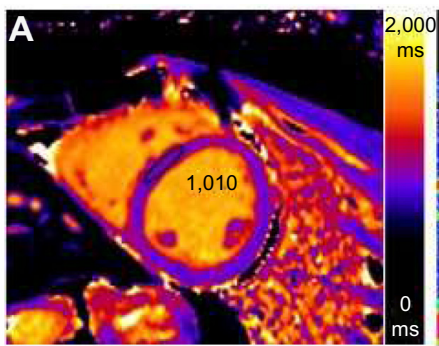

MOLLI

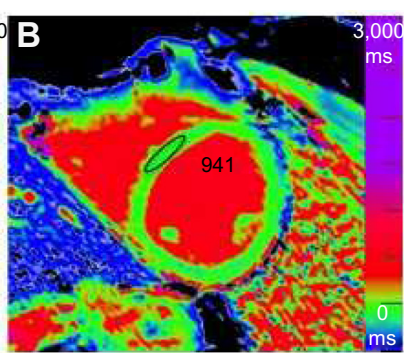

ShMOLLI

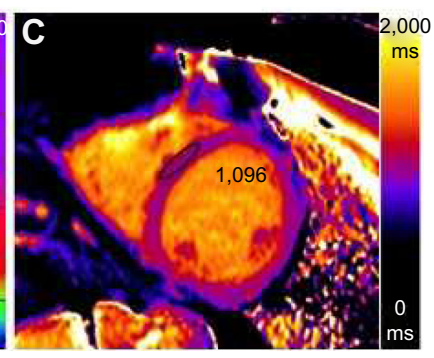

SASHA

Figure 2 Examples of native TI maps from a healthy volunteer using three different sequences.

Notes: (A) MOLLI, (B) ShMOLLI, and (C) SASHA, each demonstrating regions of interest and their values. Note the difference in values. MOLLI and ShMOLLI can underestimate native myocardial TI in comparison to a standard spin echo acquisition, whereas SASHA yields a higher accuracy and lower precision compared with MOLLI and ShMOLLI.

Abbreviations: MOLLI, modified Look-Locker inversion recovery; SASHA, saturation recovery single-shot acquisition; ShMOLLI, shortened MOLLI. 
systematically underestimate native myocardial T1 in comparison to standard spin echo acquisition, whereas SASHA and SAPPHIRE yield higher accuracy, lower precision, and similar reproducibility compared with MOLLI and ShMOLLI for T1 measurement. ${ }^{19-21}$ In practice, this means that different techniques measure different normal values (Figure 2).

\section{Native TI mapping}

Native T1 mapping (pre- or non-contrast T1) changes with disease (Figure 3). T1 values are higher with expansion of the extracellular compartment by fibrosis, ${ }^{22}$ edema, ${ }^{23}$ and amyloid (Figure 3B), ${ }^{24}$ and lower in lipid accumulation (Anderson-Fabry disease) (Figure 3D), ${ }^{25}$ cardiac siderosis (Figure 3C), ${ }^{26}$ and hemorrhage. ${ }^{27}$ These changes may be detectable in early disease, may be robust enough to track therapy, and have been shown to be prognostic in some diseases. ${ }^{28}$ There are, however, limitations: native T1 involves measurement of a composite signal from both the interstitium and from myocytes, so the signal requires clinical interpretation and has the potential for pseudonormalization (plausible examples include fibrosis canceling out mild iron overload in sickle cell disease, or scar in Anderson-Fabry disease canceling out the fat signal). ${ }^{29}$ As mentioned above, native $\mathrm{T} 1$ values also vary with the sequence used, field strength, and the manufacturer of the magnet, highlighting the importance of obtaining normal reference ranges for each $\mathrm{CMR}$ center. ${ }^{30}$

\section{ECV fraction}

The extracellular matrix is a dynamic, complex milieu made up of hydrated collagen and other macromolecules and serves to anchor myocytes, store energy, align contractile elements, and provide a protective role by preventing overextension and disruption of myocytes. ${ }^{31}$ Expansion of the extracellular space occurs with focal fibrosis, diffuse fibrosis, edema, and infiltrative pathologies (eg, amyloidosis). Myocardial biopsy is the gold-standard method for quantifying fibrosis, but carries significant morbidity and mortality, and sampling error confounds accuracy. ${ }^{32}$ Noninvasive
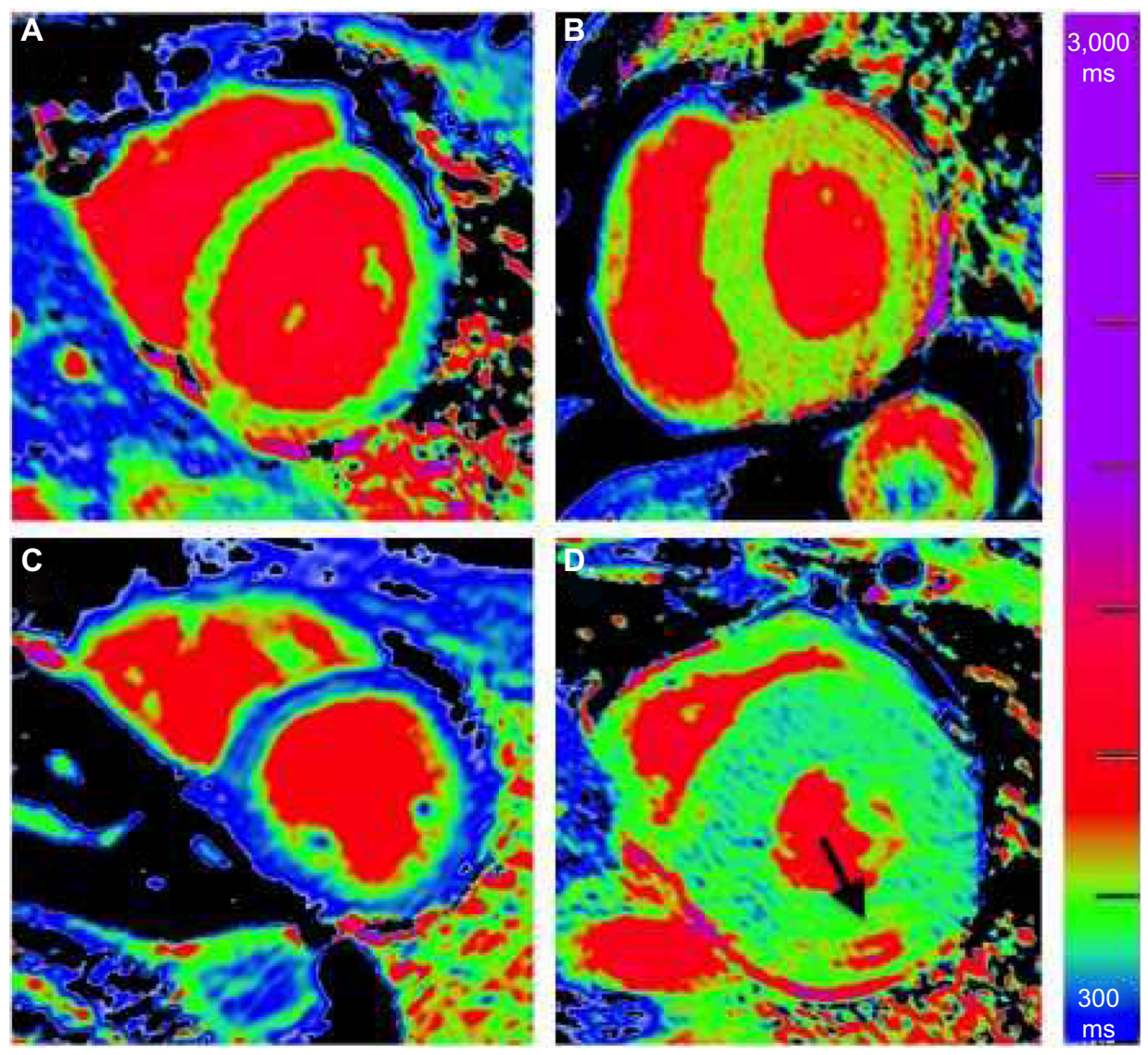

ms

Figure 3 Native TI maps in the basal short axis.

Notes: (A) Healthy volunteer. The myocardium appears homogeneously green and the blood is red. (B) Cardiac amyloid. The myocardium has a higher TI value (red). (C) Severe iron overload. The myocardium appears blue as the TI value is low from iron. (D) Anderson-Fabry disease. The myocardium has a lower TI value (blue) due to intracellular lipid accumulation, except in the inferolateral wall, which is red due to fibrosis (black arrow). 
measurement of focal fibrosis is possible with LGE imaging by CMR, but this is unable to quantify diffuse fibrosis. ${ }^{32}$ Initial methods to detect diffuse fibrosis involved measuring post-contrast T1 values after bolus extracellular contrast. ${ }^{33}$ This method, however, presented some challenges. The value is affected by renal clearance of contrast, contrast dose, body composition, timings of acquisition postcontrast, and the measured hematocrit. Measurement of the ECV fraction is preferred, as it closely reflects the amount of diffuse fibrosis. ${ }^{34}$

ECV measurement is based on three elements: 1) CMR T1 measurement of blood and myocardium pre-contrast; 2) CMR T1 measurement of blood and myocardium after a contrast bolus, allowing sufficient time to equilibrate, or after a continuous infusion; and 3) a blood test to measure blood contrast volume of distribution (1 minus the hematocrit). As there is contrast equilibrium, the ratio of signal change in blood and myocardium is the ratio of the myocardial extracellular volume fraction to blood plasma volume fraction. The ECV, which can also be computed as a pixel-by-pixel map (Figure 2C), is calculated, therefore, as:

$$
\mathrm{ECV}=(1-\text { hematocrit }) \times \frac{\left(1 / \mathrm{T} 1_{\text {myocardium post }}\right)-\left(1 / \mathrm{T} 1_{\text {myocardium pre }}\right)}{\left(1 / \mathrm{T} 1_{\text {blood post }}\right)-\left(1 / \mathrm{T} 1_{\text {blood pre }}\right)}(1)
$$

\section{Intracellular volume fraction}

Intracellular volume (ICV) $(=1-\mathrm{ECV})$ represents the myocardium not accessible to the extracellular contrast agent. Accordingly, ICV represents an intact myocardial cellular component providing a way to measure the myocytes cell volume (= ICV $\times$ left ventricular $(\mathrm{LH})$ mass). Again, it is necessary to clarify that there is a bias because, even if ICV mainly represents myocytes, it also includes fibroblasts, red blood cells, and macrophages. ${ }^{11}$

\section{Clinical utility of T I mapping} Non-fibrosis imaging: amyloid, AndersonFabry disease, iron overload, myocarditis Cardiac amyloidosis

Cardiac involvement is a major cause of morbidity and mortality in patients with amyloidosis. Accumulation of amyloid protein in the myocardial interstitium can be detected by CMR LGE and matches the distribution of amyloid on histology. ${ }^{35,36}$ This characteristic pattern may, however, occur late in the disease process and does not quantify the amyloid burden. Native T1 mapping and ECV quantification may have a high diagnostic accuracy for detecting cardiac amyloid, and are potentially more sensitive for detecting early disease. Amyloid light-chain (AL) amyloidosis patients with cardiac involvement $(n=53)$ have been shown to have significantly elevated native T1 mapping values compared with normal subjects. Values were increased even when cardiac amyloid involvement was uncertain, and correlated with markers of systolic and diastolic dysfunction. ${ }^{24}$ Mean ECV was also found to be significantly greater in AL amyloidosis $(\mathrm{n}=60)$ than in healthy controls $(0.25$ versus $0.40, P<0.001)$ and correlated with cardiac parameters by echocardiography. ${ }^{37}$ Similar native T1 findings have also been detected in transthyretin amyloidosis, but with less maximal T1 elevation. ${ }^{38}$

\section{Anderson-Fabry disease}

Anderson-Fabry disease is a rare, multi-system lipid storage disorder caused by a deficiency of $\alpha$-galactosidase A. Left ventricular hypertrophy ( $\mathrm{LVH}$ ) is the most common cardiac manifestation (Figure 4A), followed by arrhythmias and valvular disease. ${ }^{39}$ Treatment has been shown to reverse or slow at least some types of disease progression when initiated before reversible end-organ damage

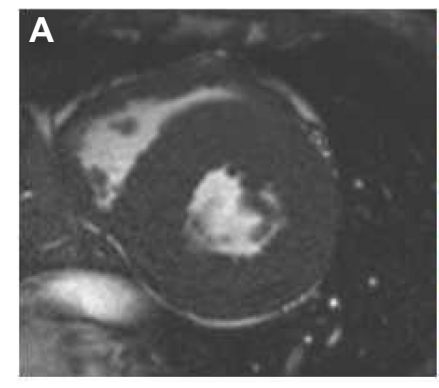

AFD SSFP cine

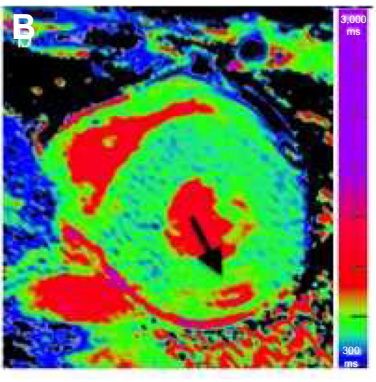

AFD ShMOLLI

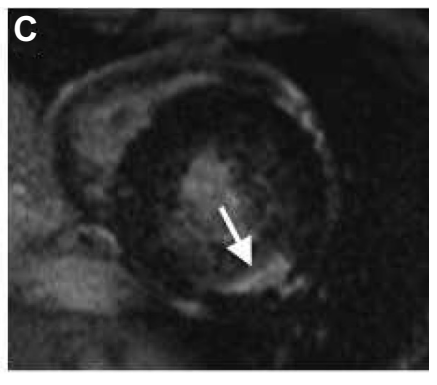

AFD LGE

Figure 4 Anderson-Fabry disease.

Notes: (A) A short-axis SSFP cine imaging showing severe concentric hypertrophy. (B) Native TI value map (ShMOLLI) demonstrating low TI (blue myocardium) with an area of fibrosis (black arrow) in the inferolateral wall. (C) LGE image demonstrating scar in the same region (white arrow).

Abbreviations: AFD, Anderson-Fabry disease; LGE, late gadolinium enhancement; ShMOLLI, shortened modified Look-Locker inversion recovery; SSFP, steady-state free precession. 
has occurred. Early detection may therefore be essential. Sado et $\mathrm{al}^{25}$ were the first to demonstrate the potential use of native T1 mapping. Compared with healthy volunteers, septal T1 was lower in Anderson-Fabry disease

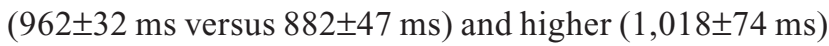
in other diseases that cause LVH $(P<0.0001)$. In this first study, T1 discriminated completely between AndersonFabry disease and other diseases with hypertrophy with no overlap. Characteristically, focal fibrosis in the basal inferolateral segments can be seen on native T1 and LGE imaging (Figure 4B and C).

\section{Iron overload}

Cardiac and liver iron deposition in primary and secondary hemochromatosis can be lethal. Treatment with aggressive chelation therapy can prevent death, but can cause serious side effects. ${ }^{40}$ There has been a marked improvement in the survival of patients with cardiac siderosis with the introduction of the CMR T2* imaging technique. ${ }^{41}$ However, the $\mathrm{T} 2 *$ sequence requires a long breath hold, and post-processing is perceived as complex. Iron also alters native T1, which is lower in iron loading. Preliminary data have shown that T1 mapping may be more sensitive and reproducible than $\mathrm{T} 2 *$ in the detection of myocardial iron. ${ }^{26}$

\section{Myocarditis}

The diagnosis of myocarditis can be challenging. Although LGE CMR is useful in the diagnosis of acute myocarditis (as part of a troponin rise and normal coronary arteries on angiography scenario), there are some recognized limitations. Patients with global edema, rather than focal areas of necrosis, may have negative findings on LGE CMR. ${ }^{42}$ Although conventional T2-weighted imaging for edema can be used, it is limited by image quality and interpretation, and reference to skeletal muscle may be confounded if that is also inflamed. ${ }^{43,44}$ Native T1 may play a key role in detecting subtle focal pathology. Ferreira et al $(n=50)$ demonstrated the superior diagnostic performance of native T1 mapping and higher sensitivity for detection of acute myocarditis and larger affected areas compared with T2-weighted and LGE CMR. ${ }^{45}$ The same group also showed that T1 mapping, in patients within 3 days of presentation, displayed typical nonischemic patterns without the need for contrast agents. ${ }^{46}$ Edema signals are increasingly being seen by native T1 mapping in rheumatic diseases, such as systemic lupus erythematosus - evidence of unrecognized myocardial involvement. ${ }^{47}$

\section{Diffuse fibrosis: valvular heart disease, heart failure, cardiomyopathy, and arrhythmias}

\section{Aortic stenosis}

Current guidelines for managing aortic stenosis (AS) have evolved from clinical assessment and measurement of valve orifice area to hemodynamic parameters such as peak velocity and mean gradient. ${ }^{48,49}$ These parameters, however, poorly predict symptom development and/or optimal timing of surgery. ${ }^{50}$ Myocardial adaptive changes are thought to play a key role in functional deterioration, symptom development, and postoperative outcome and recovery. ${ }^{51}$ Diffuse fibrosis may develop without ascribable symptoms or function changes, ${ }^{34}$ so its measurement has the potential to improve diagnostic and therapeutic management. Patients with mild-to-moderate diffuse fibrosis at baseline show improvements in symptoms and LV function and marked reduction of LVH after surgery compared with those with severe fibrosis. ${ }^{52-54}$

Bull et al $(n=109)$ were first to demonstrate increased native T1 values (by ShMOLLI) in patients with severe AS compared with controls, with correlation with fibrosis on histology. ${ }^{22} \mathrm{~A}$ further increase was seen in symptomatic patients compared with those who were asymptomatic ( $1,014 \pm 38 \mathrm{~ms}$ versus $972 \pm 33 \mathrm{~ms})$.

In patients with severe AS, ECV was also found to be persistently elevated 6 months after aortic valve replacement despite LVH regression, suggesting early LVH regression involves a cellular process. ${ }^{34}$ Multiple prospective cohort studies are currently underway to investigate the role of T1 mapping and ECV as predictive markers in AS (ClinicalTrials.gov identifiers: NCT01658345, NCT02174471, and NCT01755936). ${ }^{55}$

\section{Heart failure}

Heart failure is a major and growing cause of morbidity and mortality. Over the last 10 years, new therapies have been few and far between. A better understanding of heart failure pathophysiology and "splitting" of subtypes with more focused therapy may be needed with renewed focus on the myocardial processes directly. Standard LGE imaging has established itself as the gold standard for scar detection in heart failure. T1 mapping adds diagnostic advantages to LGE; firstly, by rare disease detection (iron, fat, amyloid), and secondly, by ECV and ICV measurements, thus allowing us to dichotomize the myocardium into its cellular $(1-\mathrm{ECV}=$ ICV) and extracellular components (diffuse fibrosis) with the high potential to impact our understanding of heart failure. 
Iles et al have shown that post-contrast T1 values reflect diffuse fibrosis histologically in patients with symptomatic heart failure $(R=-0.7, P=0.03) .{ }^{33}$ In the same patient cohort, the post-contrast $\mathrm{T} 1$ values were shorter in patients than in controls (383 $\pm 17 \mathrm{~ms}$ versus $564 \pm 23 \mathrm{~ms}, P<0.0001)$. Additionally, the post-contrast $\mathrm{T} 1$ values shortened as diastolic function worsened $(P<0.001)$. Ellims et al explored the relationship between T1 mapping and diastolic dysfunction further. ${ }^{56}$ Twenty cardiac transplant patients underwent a post-contrast myocardial T1 CMR and invasive LV pressurevolume measurements. The post-contrast T1 value and ECV correlated with LV stiffness $(r=-0.71, P=0.001$ and $r=0.58$, $P=0.04$, respectively). These findings may further enhance our understanding of the pathophysiology of the different cardiomyopathies. ${ }^{56}$

The association of post-contrast T1 mapping with prognostic outcome in heart failure with preserved ejection fraction was demonstrated by Mascherbauer et al. ${ }^{57}$ Patients with T1 values less than $388.3 \mathrm{~ms}$ were at greatest risk of cardiac events compared with the rest of the group $(P<0.01)$, thus suggesting the possible role of post-contrast T1 as a prognostic biomarker. Modest ECV changes also appear prognostic. Wong et al showed, in 793 consecutive patients followed over 1 year (excluding amyloid and hypertrophic cardiomyopathy, measuring outside LGE areas), that global ECV predicted short-term mortality. ${ }^{10}$ The same group also found $(n \sim 1,000)$ that higher ECV values in diabetic patients were associated with adverse outcome, including mortality and heart failure hospitalization. Interestingly, lower ECV values were found in patients on drugs blocking the renin-angiotensinaldosterone system. ${ }^{58}$

\section{Cardiomyopathy}

CMR has played an important role in the diagnosis of cardiomyopathy. Scar burden quantified by LGE has become a prognostic marker of patient outcome, with significant relationships seen between LGE and cardiovascular mortality, heart failure death, and all-cause mortality. ${ }^{8}$ Initial descriptions of nonischemic LGE patterns were followed by demonstrations of histological correlations. ${ }^{59-61}$ A diffuse pattern of fibrosis has now been recognized histologically which cannot be detected by LGE sequences. ${ }^{32}$ The potential use of native and post-contrast T1 mapping and ECV in the diagnosis of ischemic and nonischemic cardiomyopathies is emerging in the literature. Puntmann et $\mathrm{al}^{62}$ demonstrated a clear difference in $\mathrm{T} 1$ (native and post-contrast) and ECV values between patients with hypertrophic cardiomyopathy and nonischemic dilated cardiomyopathy compared with control subjects $(P<0.01)$. Furthermore, native T1 mapping could differentiate between the groups with high sensitivity and specificity, although post-contrast T1 and ECV values were less discriminatory. ${ }^{62}$ The measurement of ECV has also been suggested as a risk stratification tool for patients with muscular dystrophy, who are at risk of death from dilated cardiomyopathy. ${ }^{63}$ Global and regional myocardial ECV values were significantly higher in muscular dystrophy patients with cardiac involvement compared with patients without cardiac involvement and controls. ${ }^{63}$

The use of T1 mapping in the assessment of rejection in heart transplant patients and those at risk of uremic cardiomyopathy in chronic kidney disease has been explored but requires further validation. ${ }^{64,65}$

Among ongoing studies, HCMR - Novel Markers of Prognosis in Hypertrophic Cardiomyopathy, is aiming to use T1 mapping in a total outcome study of 2,750 patients (ClinicalTrials.gov identifier: NCT01915615).

\section{Arrhythmias}

Diffuse atrial and ventricular fibrosis is a well-recognized finding in patients with atrial fibrillation (AF). ${ }^{66,67}$ Surgical biopsies and autopsy samples have shown an increased amount of fibrosis in patients with AF compared with those in sinus rhythm. ${ }^{68}$ Quantification may therefore provide an insight into remodeling processes, play a role in identifying patients who could benefit from early ablation procedures, and predict procedure success. Atrial post-contrast T1 values have been shown to be significantly shorter in patients with AF compared with healthy controls, and correlate with clinical outcome following catheter ablation. Ling et al showed that post-contrast atrial T1 times $>230 \mathrm{~ms}$ were associated with a successful outcome in $85 \%$ of patients, compared with $62 \%$ with atrial T1 times $<230 \mathrm{~ms}(P=0.01) .{ }^{69}$ The same group also demonstrated significantly different ventricular post-contrast $\mathrm{T} 1$ values in patients with persistent $\mathrm{AF}$, paroxysmal AF, and healthy volunteers $(360 \pm 84 \mathrm{~ms}$ versus $427 \pm 95 \mathrm{~ms}$ versus $535 \pm 86 \mathrm{~ms}$, respectively, $P<0.001){ }^{70}$ There are two possible suggestions to account for this finding: 1) diffuse fibrosis may be a consequence of AF and tachycardia-mediated cardiomyopathy; or 2) there may be an underlying cardiomyopathy that precedes and contributes to the development of AF. ${ }^{70}$ The findings of diffuse LV fibrosis have also been documented in patients 5 years post-curative ablation, thus possibly providing a substrate for ventricular arrhythmias. $^{71}$ 


\section{Challenges for TI mapping in clinical practice}

Despite its potential, there are substantial challenges in delivering T1 mapping to clinical practice. These include quality control, standardization, normative values, regulatory environment, and stability of techniques over time. The (first) $\mathrm{T} 1$ consensus statement roadmaps some of these issues, ${ }^{11}$ aiming to achieve a balance between ongoing development of T1 mapping that is good enough for rollout and service use. The recommendation for application of T1 mapping in clinical practice and research focus on site preparation (representative local normal values for every scanner), scan acquisition (consistent parameters across pre- and postcontrast maps), quality control (use of parametric error maps), analysis (avoidance of partial voluming of blood by conservative drawing of regions of interest), and technical development (methodological validation of sequences; unique naming of new sequences; systematic testing of accuracy and precision).

\section{Future prospects}

The T1 mapping field is rapidly progressing. New technical developments are simpler to apply and more robust. ECV mapping is now a reality, and new challenges are being considered, such as whole-heart and right ventricle coverage with higher spatial and temporal resolution, advanced image registration, and new techniques to overcome or account for partial voluming of blood. Systems approaches will also be key to delivery.

\section{Conclusion}

CMR with the LGE imaging method is the gold standard for noninvasive myocardial tissue characterization and scar imaging/quantification. T1 mapping takes this forward to the measurement of diffuse processes in a quantitative pixel-by-pixel approach. Native T1 allows diagnosis of cardiac infiltration, lipid storage disease, iron overload, and edema, as well as focal and (to a lesser extent) diffuse fibrosis. ECV quantification can dichotomize the myocardium into its cellular and interstitial components. This will further our understanding of pathological mechanisms in the myocardium and should lead to better diagnostic pathways, improved prognostication, and improved monitoring of therapy.

\section{Acknowledgments}

A Abdel-Gadir is supported by a grant from the Rosetrees Trust. TA Treibel is supported by a grant from The National
Institute for Health Research (Doctoral Research Training Fellowship: DRF-2013-06-102). JC Moon is supported by the Higher Education Funding Council for England. This work was undertaken at the University College London Hospital and University College London, which received a proportion of funding from the Department of Health's National Institute for Health Research Biomedical Research Centres funding scheme.

\section{Disclosure}

The authors report no conflicts of interest in this work.

\section{References}

1. Kramer CM, Barkhausen J, Flamm SD, Kim RJ, Nagel E; Society for Cardiovascular Magnetic Resonance Board of Trustees Task Force on Standardized Protocols. Standardized cardiovascular magnetic resonance imaging (CMR) protocols, society for cardiovascular magnetic resonance: board of trustees task force on standardized protocols. J Cardiovasc Magn Reson. 2008;10:35.

2. Greenwood JP, Maredia N, Younger JF, et al. Cardiovascular magnetic resonance and single-photon emission computed tomography for diagnosis of coronary heart disease (CE-MARC): a prospective trial. Lancet. 2012;379:453-460.

3. Assomull RG, Prasad SK, Lyne J, et al. Cardiovascular magnetic resonance, fibrosis, and prognosis in dilated cardiomyopathy. $J \mathrm{Am}$ Coll Cardiol. 2006;48:1977-1985.

4. Jiji RS, Kramer CM. Cardiovascular magnetic resonance: applications in daily practice. Cardiol Rev. 2011;19:246-254.

5. Vignaux O. Cardiac sarcoidosis: spectrum of MRI features. AJR Am J Roentgenol. 2005;184:249-254.

6. Moon JC, McKenna WJ, McCrohon JA, Elliott PM, Smith GC, Pennell DJ. Toward clinical risk assessment in hypertrophic cardiomyopathy with gadolinium cardiovascular magnetic resonance. J Am Coll Cardiol. 2003;41:1561-1567.

7. Karamitsos TD, Neubauer S. The prognostic value of late gadolinium enhancement CMR in nonischemic cardiomyopathies. Curr Cardiol Rep. 2013;15:326.

8. Green JJ, Berger JS, Kramer CM, Salerno M. Prognostic value of late gadolinium enhancement in clinical outcomes for hypertrophic cardiomyopathy. JACC Cardiovasc Imaging. 2012;5: $370-377$.

9. Wu KC, Weiss RG, Thiemann DR, et al. Late gadolinium enhancement by cardiovascular magnetic resonance heralds an adverse prognosis in nonischemic cardiomyopathy. J Am Coll Cardiol. 2008;51: 2414-2421.

10. Wong TC, Piehler K, Meier CG, et al. Association between extracellular matrix expansion quantified by cardiovascular magnetic resonance and short-term mortality. Circulation. 2012;126:1206-1216.

11. Moon JC, Messroghli DR, Kellman P, et al; Society for Cardiovascular Magnetic Resonance Imaging; Cardiovascular Magnetic Resonance Working Group of the European Society of Cardiology. Myocardial T1 mapping and extracellular volume quantification: a Society for Cardiovascular Magnetic Resonance (SCMR) and CMR Working Group of the European Society of Cardiology consensus statement. J Cardiovasc Magn Reson. 2013;15:92.

12. Piechnik SK, Ferreira VM, Lewandowski AJ, et al. Normal variation of magnetic resonance $\mathrm{T} 1$ relaxation times in the human population at 1.5 T using ShMOLLI. J Cardiovasc Magn Reson. 2013;15:13.

13. Messroghli DR, Radjenovic A, Kozerke S, Higgins DM, Sivananthan MU, Ridgway JP. Modified Look-Locker inversion recovery (MOLLI) for high-resolution T1 mapping of the heart. Magn Reson Med. 2004;52:141-146. 
14. Messroghli DR, Greiser A, Fröhlich M, Dietz R, Schulz-Menger J. Optimization and validation of a fully-integrated pulse sequence for modified look-locker inversion-recovery (MOLLI) T1 mapping of the heart. J Magn Reson Imaging. 2007;26:1081-1086.

15. Piechnik SK, Ferreira VM, Dall'Armellina E, et al. Shortened Modified Look-Locker Inversion recovery (ShMOLLI) for clinical myocardial T1-mapping at 1.5 and $3 \mathrm{~T}$ within a 9 heartbeat breathhold. J Cardiovasc Magn Reson. 2010;12:69.

16. Chow K, Flewitt JA, Green JD, Pagano JJ, Friedrich MG, Thompson RB. Saturation recovery single-shot acquisition (SASHA) for myocardial T(1) mapping. Magn Reson Med. 2014;71:2082-2095.

17. Kellman P, Hansen MS. T1-mapping in the heart: accuracy and precision. J Cardiovasc Magn Reson. 2014;16:2.

18. Higgins DM, Moon JC. Review of T1 mapping methods: comparative effectiveness including reproducibility issues. Curr Cardiovasc Imaging Rep. 2014; 7:9252.

19. Rogers T, Dabir D, Mahmoud I, et al. Standardization of T1 measurements with MOLLI in differentiation between health and disease - the ConSept study. J Cardiovasc Magn Reson. 2013;15:78.

20. Messroghli DR, Plein S, Higgins DM, et al. Human myocardium: single-breath-hold MR T1 mapping with high spatial resolution reproducibility study. Radiology. 2006;238:1004-1012.

21. Roujol S, Weingärtner S, Foppa M, et al. Accuracy, precision, and reproducibility of four T1 mapping sequences: a head-to-head comparison of MOLLI, ShMOLLI, SASHA, and SAPPHIRE. Radiology. Epub April 4, 2014.

22. Bull S, White SK, Piechnik SK, et al. Human non-contrast T1 values and correlation with histology in diffuse fibrosis. Heart. 2013;99:932-937.

23. Ferreira VM, Piechnik SK, Dall'Armellina E, et al. Non-contrast T1-mapping detects acute myocardial edema with high diagnostic accuracy: a comparison to T2-weighted cardiovascular magnetic resonance. J Cardiovasc Magn Reson. 2012;14:42.

24. Karamitsos TD, Piechnik SK, Banypersad SM, et al. Noncontrast T1 mapping for the diagnosis of cardiac amyloidosis. JACC Cardiovasc Imaging. 2013;6:488-497.

25. Sado DM, White SK, Piechnik SK, et al. Identification and assessment of Anderson-Fabry disease by cardiovascular magnetic resonance noncontrast myocardial T1 mapping. Circ Cardiovasc Imaging. 2013;6:392-398.

26. Sado D, White SK, Piechnik SK, et al. Native T1 lowering in iron overload and Anderson Fabry disease; a novel and early marker of disease. J Cardiovasc Magn Reson. 2013;15(Suppl 1):O71.

27. Pedersen SF, Thrysøe SA, Robich MP, et al. Assessment of intramyocardial hemorrhage by $\mathrm{T} 1$-weighted cardiovascular magnetic resonance in reperfused acute myocardial infarction. J Cardiovasc Magn Reson. 2012;14:59.

28. Banypersad SM, Fontana M, Maestrini V, et al. Cardiac amyloid burden assessment by $\mathrm{T} 1$ mapping predicts survival in patients with systemic AL amyloidosis - a 2 year follow-up study. J Cardiovasc Magn Reson. 2014;16(1):O5.

29. Jellis CL, Kwon DH. Myocardial T1 mapping: modalities and clinical applications. Cardiovasc Diagn Ther. 2014;4:126-137.

30. Raman FS, Kawel-Boehm N, Gai N, et al. Modified look-locker inversion recovery $\mathrm{T} 1$ mapping indices: assessment of accuracy and reproducibility between magnetic resonance scanners. J Cardiovasc Magn Reson. 2013;15:64.

31. Brown L. Cardiac extracellular matrix: a dynamic entity. Am J Physiol Heart Circ Physiol. 2005;289:H973-H974.

32. Mewton N, Liu CY, Croisille P, Bluemke D, Lima JA. Assessment of myocardial fibrosis with cardiovascular magnetic resonance. $\mathrm{J} \mathrm{Am} \mathrm{Coll}$ Cardiol. 2011;57:891-903.

33. Iles L, Pfluger H, Phrommintikul A, et al. Evaluation of diffuse myocardial fibrosis in heart failure with cardiac magnetic resonance contrastenhanced T1 mapping. J Am Coll Cardiol. 2008;52:1574-1580.
34. Flett AS, Sado DM, Quarta G, et al. Diffuse myocardial fibrosis in severe aortic stenosis: an equilibrium contrast cardiovascular magnetic resonance study. Eur Heart J Cardiovasc Imaging. 2012;13:819-826.

35. Maceira AM, Joshi J, Prasad SK, et al. Cardiovascular magnetic resonance in cardiac amyloidosis. Circulation. 2005;111:186-193.

36. Cheng AS, Banning AP, Mitchell AR, Neubauer S, Selvanayagam JB. Cardiac changes in systemic amyloidosis: visualisation by magnetic resonance imaging. Int $J$ Cardiol. 2006;113:E21-E23.

37. Banypersad SM, Sado DM, Flett AS, et al. Quantification of myocardial extracellular volume fraction in systemic AL amyloidosis: an equilibrium contrast cardiovascular magnetic resonance study. Circ Cardiovasc Imaging. 2013;6:34-39.

38. Fontana M, Banypersad SM, Treibel TA, et al. Native T1 mapping in transthyretin amyloidosis. JACC Cardiovasc Imaging. 2014;7: $157-165$.

39. O'Mahony C, Elliott P. Anderson-Fabry disease and the heart. Prog Cardiovasc Dis. 2010;52:326-335.

40. Poggiali E, Cassinerio E, Zanaboni L, Cappellini MD. An update on iron chelation therapy. Blood Transfus. 2012;10:411-422.

41. Modell B, Khan M, Darlison M, Westwood MA, Ingram D, Pennell DJ. Improved survival of thalassaemia major in the UK and relation to T2* cardiovascular magnetic resonance. J Cardiovasc Magn Reson. 2008; $10: 42$.

42. Friedrich MG, Marcotte F. Cardiac magnetic resonance assessment of myocarditis. Circ Cardiovasc Imaging. 2013;6:833-839.

43. Park CH, Choi EY, Greiser A, Paek MY, Hwang SH, Kim TH. Diagnosis of acute global myocarditis using cardiac MRI with quantitative t1 and $\mathrm{t} 2$ mapping: case report and literature review. Korean $J$ Radiol. 2013;14:727-732.

44. Verhaert D, Thavendiranathan P, Giri S, et al. Direct T2 quantification of myocardial edema in acute ischemic injury. JACC Cardiovasc Imaging. 2011;4:269-278.

45. Ferreira VM, Piechnik SK, Dall'Armellina E, et al. T(1) mapping for the diagnosis of acute myocarditis using CMR: comparison to T2-weighted and late gadolinium enhanced imaging. JACC Cardiovasc Imaging. 2013;6:1048-1058.

46. Ferreira VM, Piechnik SK, Dall'Armellina E, et al. Native T1-mapping detects the location, extent and patterns of acute myocarditis without the need for gadolinium contrast agents. J Cardiovasc Magn Reson. 2014;16:36.

47. Puntmann VO, D'Cruz D, Smith Z, et al. Native myocardial T1 mapping by cardiovascular magnetic resonance imaging in subclinical cardiomyopathy in patients with systemic lupus erythematosus. Circ Cardiovasc Imaging. 2013;6:295-301.

48. Bonow RO, Carabello BA, Chatterjee K, et al; American College of Cardiology/American Heart Association Task Force on Practice Guidelines. 2008 focused update incorporated into the ACC/AHA 2006 guidelines for the management of patients with valvular heart disease: a report of the American College of Cardiology/American Heart Association Task Force on Practice Guidelines (Writing Committee to revise the 1998 guidelines for the management of patients with valvular heart disease). Endorsed by the Society of Cardiovascular Anesthesiologists, Society for Cardiovascular Angiography and Interventions, and Society of Thoracic Surgeons. J Am Coll Cardiol. 2008;52:e1-e142.

49. Taylor J. ESC/EACTS Guidelines on the management of valvular heart disease. Eur Heart J. 2012;33:2371-2372.

50. Vahanian A, Baumgartner H, Bax J, et al; Task Force on the Management of Valvular Hearth Disease of the European Society of Cardiology; ESC Committee for Practice Guidelines. Guidelines on the management of valvular heart disease: The Task Force on the Management of Valvular Heart Disease of the European Society of Cardiology. Eur Heart J. 2007;28:230-268.

51. Dweck MR, Boon NA, Newby DE. Calcific aortic stenosis: a disease of the valve and the myocardium. J Am Coll Cardiol. 2012;60: $1854-1863$ 
52. Milano AD, Faggian G, Dodonov M, et al. Prognostic value of myocardial fibrosis in patients with severe aortic valve stenosis. J Thorac Cardiovasc Surg. 2012;144:830-837.

53. Shin S, Park PW, Han WS, Sung KI, Kim WS, Lee YT. Mass reduction and functional improvement of the left ventricle after aortic valve replacement for degenerative aortic stenosis. Korean J Thorac Cardiovasc Surg. 2011;44:399-405.

54. Weidemann F, Herrmann S, Stork S, et al. Impact of myocardial fibrosis in patients with symptomatic severe aortic stenosis. Circulation. 2009; 120:577-584.

55. Singh A, Ford I, Greenwood JP, et al. Rationale and design of the PRognostic Importance of MIcrovascular Dysfunction in asymptomatic patients with Aortic Stenosis (PRIMID-AS): a multicentre observational study with blinded investigations. BMJ Open. 2013;3:e004348.

56. Ellims AH, Shaw JA, Stub D, et al. Diffuse myocardial fibrosis evaluated by post-contrast $\mathrm{t} 1$ mapping correlates with left ventricular stiffness. J Am Coll Cardiol. 2014;63:1112-1118.

57. Mascherbauer J, Marzluf BA, Tufaro C, et al. Cardiac magnetic resonance postcontrast $\mathrm{T} 1$ time is associated with outcome in patients with heart failure and preserved ejection fraction. Circ Cardiovasc Imaging. 2013;6:1056-1065.

58. Wong TC, Piehler KM, Kang IA, et al. Myocardial extracellular volume fraction quantified by cardiovascular magnetic resonance is increased in diabetes and associated with mortality and incident heart failure admission. Eur Heart J. 2014;35:657-664.

59. Moravsky G, Ofek E, Rakowski H, et al. Myocardial fibrosis in hypertrophic cardiomyopathy: accurate reflection of histopathological findings by CMR. JACC Cardiovasc Imaging. 2013;6:587-596.

60. Moon JC, Reed E, Sheppard MN, et al. The histologic basis of late gadolinium enhancement cardiovascular magnetic resonance in hypertrophic cardiomyopathy. J Am Coll Cardiol. 2004;43:2260-2264.

61. Choudhury L, Mahrholdt H, Wagner A, et al. Myocardial scarring in asymptomatic or mildly symptomatic patients with hypertrophic cardiomyopathy. J Am Coll Cardiol. 2002;40:2156-2164.
62. Puntmann VO, Voigt T, Chen Z, et al. Native T1 mapping in differentiation of normal myocardium from diffuse disease in hypertrophic and dilated cardiomyopathy. JACC Cardiovasc Imaging. 2013;6:475-484.

63. Florian A, Ludwig A, Rosch S, Yildiz H, Sechtem U, YilmazA. Myocardial fibrosis imaging based on T1-mapping and extracellular volume fraction (ECV) measurement in muscular dystrophy patients: diagnostic value compared with conventional late gadolinium enhancement(LGE) imaging. Eur Heart J Cardiovasc Imaging. 2014;15:1004-1012.

64. Vermes E, Pucheux J, Delhommais A, Alison D, Brunereau L. Cardiac magnetic resonance $\mathrm{T} 1$ mapping pre and post contrast in heart transplant patients with clinical antibody-mediated rejection: a preliminary experience. J Cardiovasc Magn Reson. 2014;16(Supp1 1):P100.

65. Edwards NC, Moody WE, Chue CD, Ferro CJ, Townend JN, Steeds RP. Defining the natural history of uremic cardiomyopathy in chronic kidney disease: the role of cardiovascular magnetic resonance. JACC Cardiovasc Imaging. 2014; 7:703-714.

66. Frustaci A, Chimenti C, Bellocci F, Morgante E, Russo MA, Maseri A. Histological substrate of atrial biopsies in patients with lone atrial fibrillation. Circulation. 1997;96:1180-1184.

67. Burstein B, Nattel S. Atrial fibrosis: mechanisms and clinical relevance in atrial fibrillation. J Am Coll Cardiol. 2008;51:802-809.

68. Beinart R, Khurram IM, Liu S, et al. Cardiac magnetic resonance T1 mapping of left atrial myocardium. Heart Rhythm. 2013;10: 1325-1331.

69. Ling LH, McLellan AJ, Taylor AJ, et al. Magnetic resonance postcontrast T1 mapping in the human atrium: validation and impact on clinical outcome following catheter ablation for atrial fibrillation. Heart Rhythm. 2014;11:1551-1559.

70. Ling LH, Kistler PM, Ellims AH, et al. Diffuse ventricular fibrosis in atrial fibrillation: noninvasive evaluation and relationships with aging and systolic dysfunction. J Am Coll Cardiol. 2012;60:2402-2408.

71. Ling LH, Kalman JM, Ellims AH, et al. Diffuse ventricular fibrosis is a late outcome of tachycardia-mediated cardiomyopathy after successful ablation. Circ Arrhythm Electrophysiol. 2013;6:697-704.
Research Reports in Clinical Cardiology

\section{Publish your work in this journal}

Research Reports in Clinical Cardiology is an international, peerreviewed, open access journal publishing original research, reports, editorials, reviews and commentaries on all areas of cardiology in the clinic and laboratory. The manuscript management system is completely online and includes a very quick and fair peer-review system.

\section{Dovepress}

Visit http://www.dovepress.com/testimonials.php to read real quotes from published authors. 